C2006 IEEE. Personal use of this material is permitted. However, permission to reprint/republish this material for advertising or promotional purposes or for creating new collective works for resale or redistribution to servers or lists, or to reuse any copyrighted component of this work in other works must be obtained from the IEEE. 


\title{
Use of Ontology Technology for Standardization of Medical Records and Dealing with Associated Privacy Issues
}

\author{
Maja Hadzic, University of Technology Sydney \\ maja.hadzic@cbs.curtin.edu.au \\ Tharam Dillon, University of Technology Sydney \\ tharam@it.uts.edu.au \\ Elizabeth Chang, School of Information Systems, Curtin University of Technology \\ elizabeth.chang@cbs.curtin.edu.au
}

\section{Abstract}

Our goal is to move towards efficient and secure use of medical records for the purpose of correct patient identification, diagnosis, appointments scheduling and the like in everyday life as well as in emergency situations.

We place emphasis on standardized use of medical records within various regions, countries and even continents. Ontologies can be used for this purpose. Instantiation of the Generic Medical Record Ontology concepts result in Specific Medical Record Ontologies that act as personalized medical records. Ontology files are machine readable and are suitable to be used within the information system. Medical record databases contain personal medical records. Through use of ontologies for standardization of medical records from these different databases, one big virtual database is created that contains medical records of all people. Another advantage of use of ontologies within the system is that hierarchical structure of ontologies will result in better control over access and use of personalized medical information addressing privacy issues associated with this.

The significance of this research lies in use of ontology technology for the purpose of establishing worldwide standardization of medical records and dealing with associated privacy issues.

\section{Introduction}

An electronic medical record (EMR) is a computer-based patient medical record. Use of EMRs has many advantages such as facilitating access to patient data by clinical staff at any given location, building automated checks for drug and allergy interactions, prescriptions, scheduling an the like [13].

National Health Service (NHS) in the United Kingdom has been carrying out one of the largest projects for a national EMR. The goal of this project is to have $60,000,000$ patients with a centralized electronic medical record by 2010 [14]. Alberta Netcare project is a large-scale operational EMR system proposed in Canada [13]. Use of electronic medical records by US doctors is increasing slowly. Less than $10 \%$ of American hospitals have implemented health information technologies [1], while about $16 \%$ of primary care physicians use electronic health records [2].

The development of standards for EMR is at the forefront of the health care agenda. EMRs need to be made interoperable to enable practicing physicians, pharmacists and hospitals to share patient information. This is 
necessary for timely, patient-centred and portable care. Currently, there are multiple competing vendors of EHR systems, each selling a software suite that is mostly not compatible with those of their competitors [13].

There are few standards for EMR systems as a whole. However, there are many standards relating to specific aspects of EHRs and EMRs. These include standards such as ASTM Continuity of Care Record. This is a patient health summary standard based upon XML. Records can be created, read and interpreted by various EHR or EMR systems [3].

Lack of a standard for interoperability among competing software options is one of the major barriers to adopting an EMR system [4]. Ontologies are a shared and formal conceptualization of a specific knowledge and can be used for data standardization [9]. In our case, ontologies can be designed to uniquely describe a personal medical record. The first aim of our project is to use ontologies in order to enable standardization of medical records data.

According to the Medical Records Institute [13], five levels of an Electronic HealthCare Record (EHCR) can be distinguished: the Automated Medical Record, the Computerized Medical Record (CMR), the Electronic Medical Record (EMR), the Electronic Patient Record (EPR) and the Electronic Health Record (EHR). As this information is interrelated and clear borders can not be drawn, we believe that medical record information should be kept in one comprehensive format. As ontologies enable information to be expressed on different levels, the second aim of this project is to use ontologies for the purpose of creating a single comprehensive formalized, standardized personal medical record that will contain all of the above mentioned information. One record can be used to uniquely decribe the health condition of a person, their medical history, appointment schedules an so forth. We believe in the global standardization of medical records. All hospitals, health institutes, patients etc. should work together in order to find mutually supportive roles and move towards this shared vision. Use of the ontologies as well as ontology-based agents within the system is discussed in Section 2.

It has been proven that effective EMR implementation and networking could save more than $\$ 81$ billion annually through improving health care efficiency and safety. Those savings could be doubled through health information technology-enabled prevention and management of chronic disease. However, this can not be realized without implementing required changes within the health care system [5]. The third aim of this project is to propose a Medical Records Information System (MRIS) which will be based on use of medical records. Its main components are discussed in Section 3. We also discuss preference of usage of medical card over VeriChip [10].

Our fourth goal is to provide an infrastructure for the MRIS so that the privacy issues associated with access and use of medical information from medical records will be addressed. This is discussed in Section 4.

\section{Use of Ontology and Onto-Agents within MRIS}

If the available information is to be unified among various medical record databases and accessed by digital components from various locations, standardization of data needs to take place. Ontologies can be used for this purpose. Moreover, the use of 
ontologies adds semantics to the model and enables meaningful interpretation of the data.

Medical Record Ontology can be used to keep personal information in a comprehensive format. Instantiation of the Generic medical record ontology concept results in Specific medical record ontologies that act as personal medical records. Personal medical records within MRIS are kept in the format of Generic medical record ontology.

In Figure 1, we show top-level hierarchy of Generic medical record ontology that can be used to represent medical and health knowledge regarding a particular person.

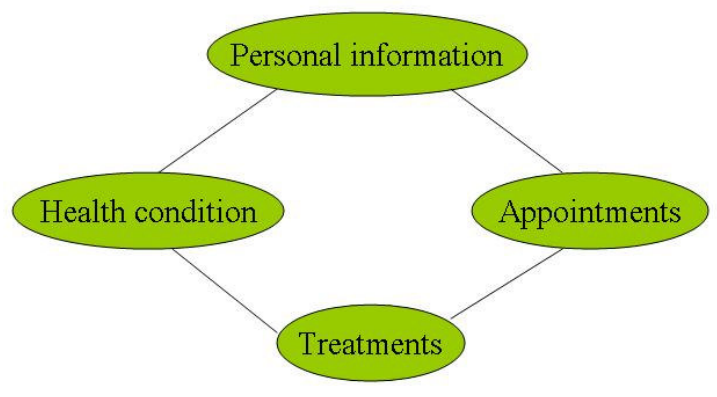

Figure 1: Four subontologies of the Generic Medical Record Ontology

We believe that four main subontologies should be created. The first subontology contains knowledge and information that will help identification of a person (personal information subontology). The second subontology contains information regarding health conditions of that particular person such as information about diseases this person is suffering from (health conditions subontology). The third subontology contains information about previous and current treatments (treatments subontology). This may be helpful in situations when a doctor is prescribing new drugs and trying to avoid drug interactions. The fourth subontology will contain information about a patient's appointments with different doctors and for different purposes. Each of the subontologies is further branched in order to precisely define the required knowledge in regard to personal information, patient's health condition, treatments and appointments. Assigning values and attributes to the concepts of the Generic medical record ontology results in Specific medical record ontologies. These Specific medical record ontologies act as personal medical records that uniquely describe health/medical conditions of a person. We propose onto-agents as ontologybased intelligent leading software species that have strong reasoning capabilities which can manage, coordinate and collaborate for the purpose of operation and maintenance of the system. Onto-agents commit to the common Generic medical record ontology. This means they obey the agreement with respect to the semantics of the concepts and relationships defined in the ontology and agree to use the shared vocabulary in a coherent and consistent manner [9]. Because ontologies are stored as machinereadable files, onto-agents can read personal records defined as ontology files and make this information available for the user. Onto-agents can also do the matching of this information with the available medical records defined as ontology files in networked medical record databases and take actions according to the results.

\section{Three Main Components of the MRIS}

We believe that the medical community can be supported through optimal use of the information 
available through machine-readable medical records. This information can be accessed through use of digital components that will screen and read the personal medical records. These personalized medical records are of the form of a medical card. Also, medical record resources that are networked within the system contain machine-readable personal medical record information and serve as a backup for this information. The format into which this information is stored is the same regardless of what region, country or continent this medical record database is situated.

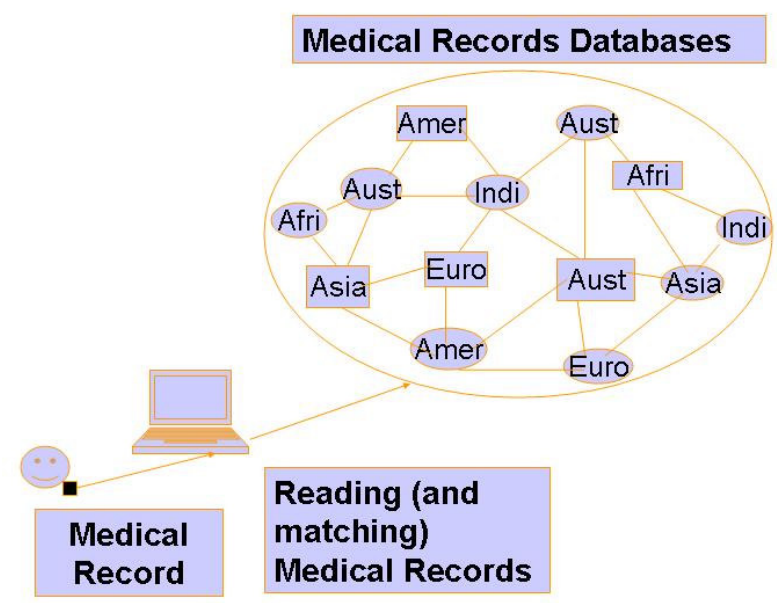

Figure 2: Medical Record Digital Components (MRDC)

We propose a Medical Record Information System (MRIS). We believe that MRIS may be prototyped on a small region but should eventually be spread globally. MRIS may contain various components but the key components should be:

- Medical record databases that contain personalized medical records;

- Screening components that read personal medical records. This information may be matched to the available information from the medical record databases; and
- Machine-readable personal medical records in the form of a medical card.

In Figure 2, we show the three main components of the MRIS. Different medical record databases from different parts of the world form a network of interrelated parts. This network is conceptually regarded as one big medical record database that contains personal medical records of all humans inhabiting our planet. The second MRIS component reads personal medical records from medical cards. This data may be matched against data from the networked medical record databases. Such screening components would need to be present in all hospitals and medical and health care institutions which would be in contact with patients. Also, emergency ambulances need to be equipped with these digital components in order to enable immediate access to patient medical record and make the most optimal decisions. Each person carries its machine-readable personal medical record in the form of a medical card. This is a third component of the MRIS. This information is read by screening components and may be matched against data from medical record databases.

Using of a medical card is preferred over Verichip technology for the following reasons [10]: VeriChip implant, marketed by the Applied Digital Company, is about the size of a grain of rice and uses radio waves to transmit medical and financial account information to reader devices. The Food and Drug Administration approved VeriChip technology. However, there are several major negative effects associated with the Verichip device. These include adverse tissue reaction, electromagnetic interference, electrical hazards, magnetic resonance imaging 
(MRI) incompatibility, failure of implanted transponder, migration of implanted transponder and so forth. Also, it is possible for the electrical currents to be induced in conductive metal implants resulting in potentially severe patient burns.

Katherine Albrecht, the founder and director of CASPIAN (Consumers Against Supermarket Privacy Invasion and Numbering), said in a statement: "By omitting this information from their press material, the companies marketing the Verichip have painted an inaccurately rosy picture of their product that could mislead consumers into believing the devices are completely safe."

In addition to outlining the health risks of the VeriChip, there is also a risk of compromised information security among its concerns. There is a risk that device transmission could be intercepted and duplicated by others or used to track an individual's movements and location. This makes VeriChip a very poor choice for medical patients seeking safety and security.

\section{Privacy Issue within the MRIS}

With guiding policy and current technology, an electronic medical record may offer better security than a traditional paper record [11]. However, the privacy issue associated with access and manipulation of personal data from medical records may not to be neglected within the MRIS. One of the ways to deal with this issue is to authorize the use of MRIS components. Only authorized persons will possess screening devices. Furthermore, access to the medical record can be limited depending on the screening purpose. For example, the system may be designed in such a way that in order to access the information represented by the four different subotologies (personal information, health condition, treatments and appointments), four different accesses are needed. Some authorized people will have access to all four subontologies through one key while others will have limited access. For example, a receptionist just needs access to the appointments subontology. Furthermore, within each subontology, access may be further limited. For example, a receptionist does not need to access information regarding previous appointments in order to book the next appointment. So, her/his access to the appointments ontology may be further limited. We can see from this example that the hierarchical ontology structure supports not only data standardization and structuring but also brings in a better control over authorized data access.

Connecting the electronic health record to the digital library also requires positive resolution of important policy issues including standards for health data and for digital libraries, health data privacy, electronic intellectual property rights etc. [12]. Use of the ontologies within the system addresses these issues. Similar to the example mentioned in the previous paragraph, access to the information available through medical record databases may be limited in accordance with person task within the system. Moreover, records regarding access into the system and use of the system need to be kept for referencing purposes.

\section{Discussion and Conclusions}

We proposed an ontology-based Medical Records Information System (MRIS) to provide an infrastructure for standardized, global and safe use of 
medical records. This organization network activates a virtuous circle through dynamic integration of three key components: the first component embraces different medical record databases scattered around world that contain personalized medical records; the second component reads personal records and possibly matches this data with personal records from medical records databases; and the third component contains machinereadable personalized medical records in the form of a medical identification card. We use Generic medical record ontology to keep the medical information in a comprehensive format. Instantiation of the Generic medical record ontology concepts results in Specific medical record ontologies that act as personalized medical records.

We also showed how hierarchical ontology structure helps address privacy issues associated with access and use of personal medical information. We discussed dangers associated with the use of VeriChip.

The significance of the research lies in use of ontology technology for the purpose of efficient and secure use as well as global standardization of medical records.

\section{References}

[1] Ringold, D.J., Santell, J.P. \& Schneider, P.J., 2000, 'ASHP national survey of pharmacy practice in acute care settings: dispensing and administration1999', American Journal of HealthSystem Pharmacy, vol. 57, no. 19. pp. 1759-75.

[2] Johnston, Doughlas, et al., 2003, 'The value of computerize provider order entry in ambulatory settings: Executive preview', MA: Center for Information Technology Leadership.
[3] Chheda, N.C., 2005, 'Electronic medical records and continuity of care records - The utility theory', Application of Information Technology and Economics.

[4] Gans, D., Kralewski, J., Hammons, T. \& Dowd, B., 2005, 'Medical groups' adoption of electronic health records and information systems', Health affairs (Project Hope), vol. 24, no. 5, pp. 1323-1333.

[5] Hillestad, R., Bigelow, J., Bower, A., Girosi, F., Meili, R., Scoville R. \& Taylor, R., 2005, 'Can electronic medical record systems transform health care? Potential health benefits, savings and costs', Health Affairs, vol. 24, no. 5, pp. 11031117.

[6] Dini, P. \& Nicolai, A., 'D.B.E. - The digital business ecosystem'. Retrieved: $20^{\text {th }}$ of February 2006 from http://www.digitalecosystems.org/doc/dbe_summary_ cc.pdf.

[7] European Commission (a), 'Technologies for digital ecosystems - Innovation ecosystem initiative'. Retrieved $20^{\text {th }}$ of February 2006 from http://www.digitalecosystems.org/

[8] European Commission (b), 'What is an European digital ecosystem?' Retrieved $24^{\text {th }}$ of February 2006 from

http://europa.eu.int/comm/enterprise /ict/conferences/doc/p5_de2.pdf.

[9] Gruber, T., 1995, 'Towards principles for the design of ontologies used for knowledge sharing', International Journal of Human and Computer Studies, vol. 43, no. 5-6, pp. 907-928.

[10] WorldNetDaily.com (2004), 'Implantable chip's medical dangers'. Retrieved $20^{\text {th }}$ of September 2006 from 
http://www.infowars.com/print/bb/im plantable_chips_medical.htm

[11] Barrows, R.C.Jr. \& Clayton, P.D., 1996, 'Privacy, confidentiality, and electronic medical records', Journal of American Medical Informatics Association, vol. 3, no. 2, pp. 139148.

[12] Humphreys, B.L., 2000, 'Electronic health record meets digital library: a new environment for achieving an old goal', Journal of American Medical Informatics Association, vol. 7, no. 5, pp. 444-452.

[13]http://en.wikipedia.org/wiki/Electronic_ medical_record

[14] http://www.nhs.uk/ 